\title{
Interstitial brachytherapy for penile cancer: the experience of Oncology Centre in Bydgoszcz
}

\author{
Roman Makarewicz, MD, PhD, Prof. ${ }^{12}$, Andrzej Lebioda, MD, PhD², Joanna Terlikiewicz, MD, PhD 1.2, \\ Renata Kabaciniska, MSc, PhD.2 \\ 'Brachytherapy Department, Oncology Centre in Bydgoszcz, Poland, 2Chair and Clinic of Oncology and Brachytherapy, \\ Nicolaus Copernicus University in Torun, Ludwik Rydygier Collegium Medicum in Bydgoszcz, Poland
}

\begin{abstract}
Purpose: The aim of this work was to analyze the treatment results and complications of patients with penile cancer treated in our centre.

Material and methods: Thirty three patients were included in this retrospective research. The average age was 62. All patients underwent brachytherapy HDR or PDR procedures using a remote-after-loading system with iridium source.

Results: Average follow-up was 60 months. Complete remission was observed in 24 patients $(72.7 \%)$ out of the whole group. Locoregional and distant recurrences were noted in 9 patients (27.3\%). In all patients 5-year overall and disease free survival were $84.9 \%$ and $75.4 \%$, respectively. The 5-year locoregional control rate was $78.8 \%$.

Conclusion: Radical brachytherapy could possibly be a good treatment option in selected patients with T1-3 penile cancer and lesions smaller than $4 \mathrm{~cm}$ in diameter.

Key words: HDR brachytherapy, penile cancer.

\section{Purpose}

Penis cancer represents $0.4-0.6 \%$ of all cancers cases in male population. Many epidemiological studies have documented the fact that penile cancer is more frequent in uncircumcised men with poor hygiene, phimosis and human papilloma virus infection. Although trauma and veneral diseases have been associated with penile cancer, no causative relationship has been identified [1]. The traditional surgical approach (partial or total penectomy depending on the extent of the disease) provides excellent local control. Radiotherapy of the primary tumor is an alternative organ preserving approach with good results in selected patients with $\mathrm{T}_{1-2}$ lesions with $<4 \mathrm{~cm}$ in diameter [2]. Radiation therapy can be delivered as an external beam radiation therapy (EBRT) or as brachytherapy using mainly Iridium-192 and high-dose-rate (HDR) remote afterloading technique. However, the best results have been obtained using brachytherapy with local control rates, ranging from $70 \%$ to $90 \%$. Most patients with local failure after radiation therapy can be salvaged surgically. Because of the lack of randomized trials comparing these different treatment modalities, the treatment decision is mainly based on a center's experience and international guidelines.

In this paper we report results of interstitial HDR brachytherapy in 33 men with penile squamous cell cancer.

\section{Material and methods}

From October 1996 to September 2007, 33 male patients with penile squamous cell cancer were treated using brachytherapy HDR at Oncology Centre in Bydgoszcz. The median age was 62 , ranged from 47 to 78 . The majority of tumors was found in the glands ( 28 cases); in 3 patients the tumor was located on prepuce and glands, in 2 cases on the glands and shaft. There were $23(69.7 \%)$ of $\mathrm{T}_{1}$ tumor cases, $7(21.2 \%)$ of $\mathrm{T}_{2}$ and $3(9.1 \%)$ of $\mathrm{T}_{3}$ tumor cases. In all patients the tumor size ranged from 2 to $4 \mathrm{~cm}$. Thirty patients were clinically node negative. Three patients were diagnosed with palpable inguinal lymph nodes and surgical dissection was applied. In two cases EBRT was used as an adjuvant therapy after excision of lymph nodes. Tumor grade was described as follows: 15 cases (53,6\%) with grade 1, 10 with grade $2(35.7 \%)$ and 3 cases with grade $6(10.7 \%)$. In 2 patients the gradation was not determined.

Implantation of needles was performed under general anesthesia. Needle placement required 30-45 minutes in the operating room. Before the procedure, catheterization was performed for all patients. Paris system of dosimetry was used for all implants. The system was usually slightly modified (stepping source was used) because of the flexibility in choosing dwell times that permits uniform dose distribution. The median number of needles was 4 ranged 
from 2 to 6. Plexiglas templates were used to maintain the geometry of an implant. The GTV includes all visible and palpable tumor. The CTV consists of GTV including margin of 8-10 mm surrounding clinically evident tumor. The prescribed dose ranged from 48 to $54 \mathrm{~Gy}$ with mean dose of 51 Gy given two times daily using HDR remote afterloading device (Nucletron) or $60 \mathrm{~Gy}$ applied for six consecutive days using PDR afterloader (Nucletron). The brachytherapy needles were well tolerated. During therapy that lasted less than 10 days, patients remained catheterized, being allowed out of bed since the treatment was applied for a short period of time. At the end of the treatment, the needles were removed after pre-medicating a patient with a narcotic analgesic. The median followup period was 60 months, ranged 37-168 months. The primary end point was the time to local failure, secondary endpoints - the time to regional and distant failure, disease free survival and overall survival.

The Kaplan-Meier estimation was used to evaluate the survival control rate and locoregional control rate, and survival was measured from the date of pathologic diagnosis.

\section{Results}

In the median period of 60 months we observed 9 patients relapsed: 4 cases of local failure, 2 - locoregional failure, 1 patient with locoregional/distant failure and 1 with a distant failure. Salvage penectomy or partial penectomy was performed in 5 cases with local or locoregional failure. Four $(80 \%)$ of 5 cases with local or locoregional failures were successfully treated with surgery. In all patients, 5 year overall and disease free survival rates were $84.9 \%$ (95\% CI) and 75.4\% (95\% CI), respectively (Fig. 1). The 5 years locoregional control rate was $78.8 \%$ (95\% CI) (Fig. 2).

The acute reactions after radiation were limited to the implant site and were observed in all patients. Moist desquamation peaks were observed within 1 to 2 weeks after brachytherapy and healed completely after 2-3 months. Sterile distal urethritis were noticed in 10 patients. In general, the cosmetic outcome was good to excellent. In 5 cases teleangiectasia was noticed, however its appearance was quite acceptable to the patient. Erectile functionality was

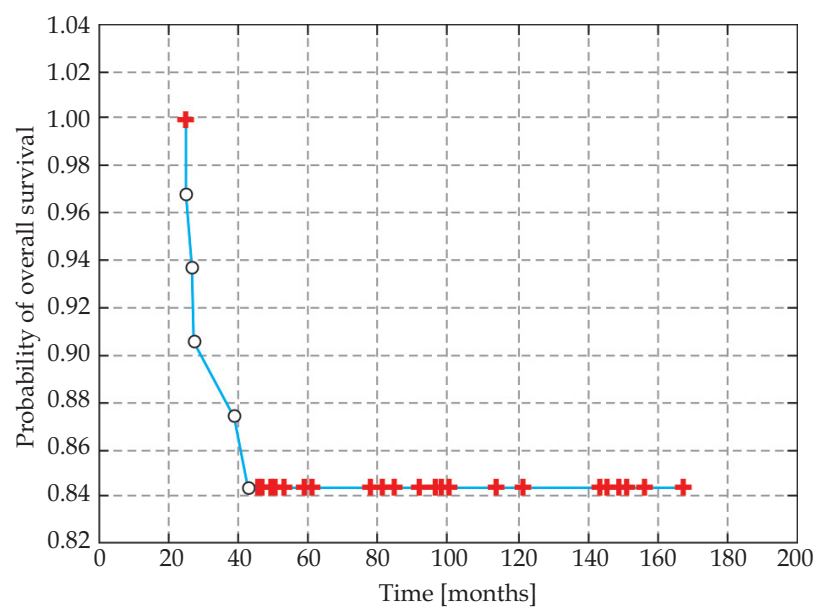

Fig. 1. Overall survival rate usually maintained at the same level as before irradiation. Soft tissue necrosis was observed in 3 patients at 10, 12 and 19 months after brachytherapy.

\section{Discussion}

Penile cancer is an uncommon disease in developed countries. More frequent occurrence of this disease is observed in developing nations [1]. The traditional policy management of penile squamous cell cancer is surgery and involves partial or total penectomy. For treatment of the primary tumor, $1-2 \mathrm{~cm}$ margin of resection is necessary to avoid an unacceptable rate of local recurrence - it is the standard of care [3]. It is understandable that local failure rate is very low after total or partial penectomy. However, psychosexual morbidity of a patient is also the subject of concern. Many conservative therapies such as laser resection, brachytherapy and EBRT are alternative modalities for a surgery and offer good local control with functional organ preservation [4]. A potential advantage of brachytherapy is the ability to avoid penile resection. Reported results for interstitial brachytherapy show 5 -years local tumor control rates ranging from $72 \%$ to $88 \%$ [1-8].

In our series, local failure was observed in seven patients. Only in three cases it was associated with regional or distant metastases. In our present series, the failure was related to the tumor grade and its size. Numerous series present poorer results for tumors greater than $4 \mathrm{~cm}$. In 5 from 2 local failures, the tumor size before treatment were greater than $4 \mathrm{~cm}$. As it was mentioned before, local control rate strongly depends on tumor size. Mazeron et al. showed local failures rate at $11 \%$ for tumors smaller than $2 \mathrm{~cm}, 26 \%$ for tumors size 2 to $4 \mathrm{~cm}$ and $50 \%$ for those greater than $4 \mathrm{~cm}$ [9]. Our results confirm this observation. In our study, brachytherapy was well tolerated and only three patients developed soft tissue necrosis. Adverse events occurred in the area receiving a very high total dose. Soft tissue necrosis was earlier reported in $23 \%$ of patients and it was more common after brachytherapy [9]. An increased risk of necrosis has been reported for doses over 60 Gy for patients with $T_{3}$ tumors and higher-volume implants $>30 \mathrm{~cm}^{3}$ [2]. The peak time for this adverse effect

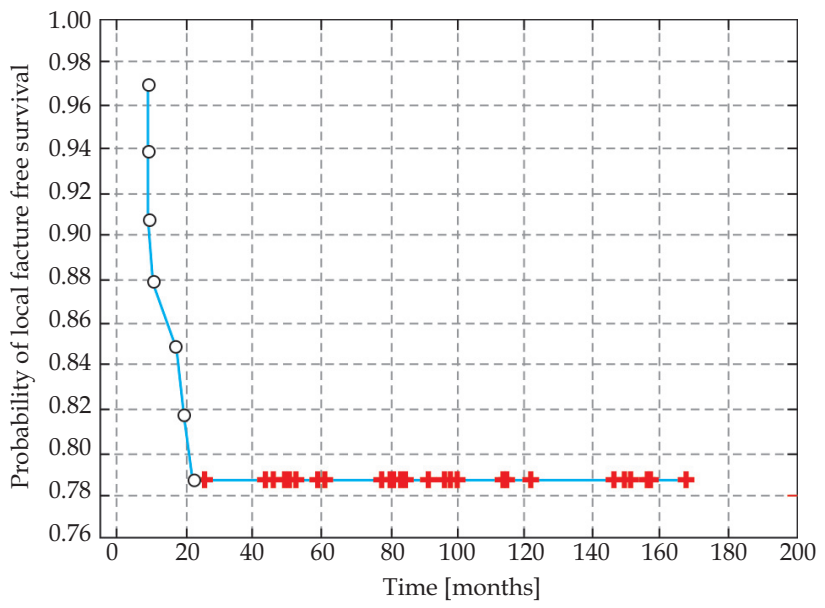

Fig. 2. Local failure free survival rate 
was 7-18 months after brachytherapy. Our series had a number of limitations. Firstly, the study group was small and it was lacking a control group for comparison. Secondly, this was a retrospective analysis and certain inherent deviation could occur.

\section{Conclusions}

Despite discussed limitations, we concluded that sole brachytherapy seems to be a satisfactory local treatment for T1-3 penile squamous cell cancer smaller than $4 \mathrm{~cm}$ in size. Penile preservation can be achieved in more than $80 \%$ of patients with no decrease in cancer control rates.

\section{References}

1. Pizzocaro G, Algaba F, Horenblas S et al. EAU penile cancer guidelines 2009. Eur Urology 2010; 57: 1002-1012.

2. Crook J, Jezioranski J, Cygler JE. Penile brachytherapy: technical aspects and postimplant issues. Brachytherapy 2010; 9: 151-158.

3. Culkin DJ, Beer TM. Advanced penile carcinoma. J Urol 2003; 170: 359-365.

4. Syed S, Eng TY, Thomas ChR et al. Current issues in the management of advanced squamous cell carcinoma of the penis. Urol Oncol: Seminars and Original Invest 2003; 21: 431-438.

5. Ozsahin M, Jichlinski P, Weber DC et al. Treatment of penile carcinoma: to cut or not to cut? Int J Radiat Oncol Biol Phys 2006; 66: 674-679.

6. Rozan R, Albuisson E, Giraud B et al. Interstitial brachytherapy for penile carcinoma: a multicentric survey (259 patients). Radioth Oncol 1995; 36: 83-93.

7. Crook JM, Jezioranski J, Math M et al. Penile brachytherapy: results for 49 patients. Int J Radiat Oncol Biol Phys 2005; 62: 460-467.

8. Crook J, Grimard L, Tsihlias J et al. Interstitial brachytherapy for penile cancer: an alternative to amputation. J Urol 2002; 167: 506-511.

9. Mazeron JJ, Lang Lois D, Lobo P. Interstitial radiation therapy for carcinoma of the pens using iridium 192 wires: the Henri Mondor experience (1970-1979). Int J Radiat Oncol Biol Phys 1984; 10: $1891-1890$. 